\title{
Quedas em idosos domiciliados e sua associação com as atividades da vida diária
}

\author{
Falls in older adults living at home and their association with daily living activities \\ Caídas sufridas por ancianos en el domicilio y su asociación con las actividades de la vida diaria
}

\author{
Rosalina Aparecida Partezani Rodrigues'; Aline Francesco da Silvall; Suzele Cristina Coelho Fabricio-Wehbe ${ }^{\text {III; }}$ \\ Marina Aleixo Diniz ${ }^{\text {IV }}$ Jack Roberto Silva Fhon ${ }^{V}$
}

\begin{abstract}
RESUMO: O objetivo deste estudo é determinar a prevalência de queda em idosos que vivem no domicílio e identificar o nível de dependência para as atividades da vida diária, entre os idosos que caíram e não caíram. Estudo transversal, com amostra por conglomerado de duplo estágio de 230 idosos, com idade acima de 70 anos, de ambos os sexos, em Ribeirão Preto, São Paulo, em 2013. Investigaram-se o perfil social, as quedas, a Escala de Lawton e Brody e o Índice de Katz. Foi identificada queda em $37,8 \%(1-7 ; \mathrm{dp}=1,0)$ dos entrevistados. Foi mais prevalente no sexo feminino $(25,2 \%)$, em idosos mais jovens (23\%). Não houve associação entre quedas e Atividades Instrumentais da Vida Diária, entretanto houve associação entre queda e Atividades Básicas da Vida Diária, pois os idosos que caíram possuíam mais dependência nestas atividades. Sugere-se que a investigação destas atividades seja utilizada como estratégia para prevenção de quedas.
\end{abstract}

Palavras-Chave: Idosos; acidentes por quedas, atividades cotidianas; enfermagem geriátrica.

\begin{abstract}
This cross-sectional study, to determine the prevalence of falls in older adults living at home, and to identify the level of dependence in activities of daily living among those who fell and those who did not, was conducted using a two-stage cluster sample of 230 individuals of both sexes, aged over 70 years, in Ribeirão Preto, São Paulo, in 2013. Social profile, falls, Lawton \& Brody scale and Katz Index were investigated. Falls were identified in $37.8 \%(1-7, \mathrm{sd}=1.0)$ of individuals interviewed. Falls were more prevalent among women $(25.2 \%)$, and younger individuals $(23 \%)$. No association was found between falls and Instrumental Activities of Daily Living, although there was an association between falls and Activities of Daily Living, as those who fell were more dependent on these activities. The study of these activities is suggested as a strategy to prevent falls. Keywords: Older adults; accidental falls; activities of daily living; geriatric nursing.
\end{abstract}

RESUMEN: El objetivo de este estudio es determinar la prevalencia de caídas sufridas por ancianos en el domicilio e identificar el nivel de dependencia para las actividades del cotidiano, entre los ancianos que sufrieron y no sufrieron caídas. Estudio transversal, con muestra por conglomerado de doble etapa de 230 ancianos, mayores de 70 años, de ambos sexos, en Ribeirão Preto, São Paulo, en 2013. Se han investigado el perfil social, las caídas, la Escala de Lawton y Brody y el Índice de Katz. Las caídas fueron identificadas en el $37,8 \%(1-7 ;$ de $=1,0)$ de los entrevistados. Hubo prevalencia en el sexo femenino $(25,2 \%)$, en ancianos más jóvenes (23\%). No existió asociación entre caídas y Actividades Instrumentales de la Vida Diaria, sin embargo, hubo asociación entre caídas y Actividades Básicas de la Vida Diaria, ya que los ancianos que se cayeron tenían mayor dependencia en estas actividades. Se sugiere que la investigación de dichas actividades se utilice como estrategia preventiva de caídas.

Palabras Claves: Ancianos; accidentes por caídas; actividades cotidianas; enfermería geriátrica.

\section{INTRODUÇÃO}

A população no mundo está envelhecendo rapidamente. Entre 2000 e 2025, a proporção de idosos maiores de 60 anos aumentará de 700 milhões para 1.200 milhões $^{1}$.

Dessa forma, o conhecimento sobre o processo de envelhecimento e a valorização de uma assistência ao idoso, voltada ao máximo para garantia de autonomia e independência e prevenção de múltiplos eventos adversos, são importantes ferramentas para um trabalho multidimensional com o idoso ${ }^{2}$.

A queda, considerada um evento adverso frequente entre idosos, merece destaque e tornou-se um

IProfessora Titular do Departamento de Enfermagem Geral e Especializada da Escola de Enfermagem de Ribeirão Preto, Universidade de São Paulo. Ribeirão Preto, São Paulo, Brasil. E-mail: rosalina@eerp.usp.br.

IIAluna de Graduação da Escola de Enfermagem de Ribeirão Preto, Universidade de São Paulo, Centro Colaborador da Organização Mundial da Saúde para o Desenvolvimento da Pesquisa em Enfermagem. Ribeirão Preto, São Paulo, Brasil. E-mail: alinefrancesco@hotmail.com.

IIIPós-Doutoranda do Departamento de Enfermagem Geral e Especializada da Escola de Enfermagem de Ribeirão Preto, Universidade de São Paulo. Ribeirão Preto, São Paulo, Brasil. Bolsista da Fundação de Amparo à Pesquisa do Estado de São Paulo. E-mail: suzelecris@ig.com.br.

IV Doutoranda do Departamento de Enfermagem Geral e Especializada da Escola de Enfermagem de Ribeirão Preto, Universidade de São Paulo. Ribeirão Preto, São Paulo, Brasil. E-mail: marinadiniz@usp.br.

vDoutorando do Departamento de Enfermagem Geral e Especializada da Escola de Enfermagem de Ribeirão Preto, Universidade de São Paulo. Ribeirão Preto, São Paulo, Brasil. E-mail: beto_fhon@hotmail.com. 
importante problema de saúde pública devido a sua alta frequência e a graves consequências que podem acarretar um elevado custo social e econômico ${ }^{2}$. Pode estar associada à diminuição da capacidade funcional do idoso, o que leva à diminuição de sua qualidade de vida.

Assim, o presente estudo tem como objetivo determinar a prevalência de queda em idosos no domicílio e o nível de dependência para as Atividades da Vida Diária (AVDs), entre os idosos que caíram e não caíram.

Com essas informações, será possível verificar se as atividades da vida diária podem ser utilizadas como ferramenta em uma avaliação geriátrica ampla para prevenção de queda. Remete à importância de se investigar a capacidade funcional do idoso mediante análise do seu desempenho na realização dessas atividades. Este estudo poderá subsidiar políticas públicas de atenção ao idoso, no que se refere à importância da prevenção de quedas, por meio da promoção da capacidade funcional dos idosos, com a garantia de autonomia e independência na realização das AVDs. Contribuirá para divulgação dos dados e conscientização dos idosos para atenção especial com sua saúde e autocuidado.

\section{REVISÃo DE LITERATURA}

A queda pode ser definida como um acontecimento involuntário em que a pessoa perde o equilíbrio e cai ao chão ou em outra superfície dura, podendo causar lesões - de leves até mortais ${ }^{3}$.

Mais de um terço dos idosos que vivem em comunidades, com idade de 65 anos ou mais, cai anualmente. Em idosos com 70 anos, esta proporção é de $32-42 \%$ e com 80 anos ou mais é de $50 \%$, percebendo-se que este evento fica mais frequente entre os idosos com o avançar da idade 4 . Ainda, um terço da população idosa sofre uma ou mais quedas por ano, sendo três vezes mais frequentes em mulheres quando comparadas aos homens; entretanto, no geral, a mortalidade associada a esta condição é maior no sexo masculino ${ }^{5}$.

Atualmente, as quedas representam 12\% das mortes no mundo entre os idosos e $40 \%$ dos óbitos por lesões nesta faixa etária. A queda é considerada a quinta causa de morte na velhice e a primeira por causa externa $^{5-7}$. Aproximadamente de 5\% a 10\% de todas as quedas em idosos que vivem na comunidade resultam em fratura e graves lesões de tecido mole e cabeça, mais de $30 \%$ passam a ter declínio em sua funcionalidade ${ }^{8-10}$.

A queda pode acarretar ao idoso vários tipos de consequências tais como, físicas - que dificultam as atividades da vida diária e com maior risco de morte; psicológicas - que podem estar relacionadas com a síndrome do medo de cair; econômicas e sociais - que geram custos para o idoso e para a sociedade e dificultam sua interação com outras pessoas fora do ambiente doméstico ${ }^{11-15}$.

Verifica-se, em prática clínica, que declínios na capacidade funcional do idoso tanto podem acarretar queda como podem ser provocados por este evento. A capacidade funcional pode ser definida como a faculdade do idoso de manter habilidades físicas e mentais necessárias para uma vida independente e autônoma, ou seja, sem necessidade de ajuda para Atividades Básicas da Vida Diária e Atividades Instrumentais da Vida Diária (ABVDs e AIVDs) ${ }^{16,17}$. Assim, sua avaliação deve ser considerada ao se planejarem ações de prevenção de queda. Manter autonomia e independência dos idosos na execução de AVDs é essencial para garantir sua qualidade de vida.

\section{Metodologia}

Trata-se de um estudo observacional e transversal, cuja amostra foi selecionada no projeto temático Condições de vida e saúde de idosos de Ribeirão Preto, São Paulo, realizado em 2008, na área urbana de Ribeirão Preto, com idosos de 65 anos ou mais que vivem na comunidade.

O processo de amostragem realizado foi probabilístico, por conglomerados e de duplo estágio. No primeiro estágio, considerou-se o setor censitário como unidade primária da amostragem (UPA). Assim, foram sorteados 30 setores censitários, com probabilidade proporcional ao tamanho de número de domicílios, entre os 600 setores do município. $\mathrm{O}$ segundo estágio foi visitar um número fixo de domicílios, com a finalidade de garantir a autoponderação amostral, sendo sorteadas a rua e a quadra onde esse processo de busca foi iniciado. Para se chegar ao número de idosos da amostra, foram visitados, no mínimo, 110 domicílios em cada setor.

Após o sorteio dos setores e identificados os bairros, foram listadas as ruas de cada setor sorteado e, posteriormente, realizado novo sorteio, determinando quais ruas deveriam ser visitadas pelos entrevistadores. Todos os idosos ausentes no domicílio, após três visitas do entrevistador, foram descartados da amostra. As entrevistas foram realizadas seguindo o sentido horário, nos quarteirões sorteados dos setores.

Ao final das 110 residências entrevistadas de cada setor, não conseguindo alcançar a densidade intradomiciliar proposta, os entrevistadores continuaram as visitas até que conseguissem o número desejado de idosos por setor.

Antes de realizar a busca pelos domicílios, os pesquisadores percorreram os setores sorteados para atualizar o número de domicílios, por setor, em 2007, 
uma vez que as informações eram referentes ao censo de 2000. Após a busca, não houve alterações significativas do número de domicílios.

Em 2008, foram entrevistados 515 idosos. No primeiro semestre de 2013, estes idosos foram contatados previamente, por telefone, para agendar uma visita para as entrevistas. Participaram da pesquisa 230 idosos. Os óbitos somaram 101(19,6\%) e perdas por mudança de endereço, recusa, institucionalização e ausência de idoso no domicílio (após três visitas), $184(35,7 \%)$.

Os dados foram coletados no domicílio dos idosos, por entrevistadores (alunos de graduação e pós-graduação) treinados, uniformizados e devidamente identificados por crachás. Foram coletadas informações pessoais e de perfil social, a partir de um instrumento desenvolvido pelo Grupo de Pesquisa em Enfermagem Geriátrica e Gerontológica da Escola de Enfermagem de Ribeirão Preto/USP (NUPEGG). A cognição foi avaliada mediante a aplicação do Miniexame do Estado Mental (MEEM), traduzido e adaptado para o Brasil ${ }^{18}$, cujo escore varia de 0 a 30 pontos. Para a análise dos dados, esta variável foi dicotomizada em sem e com déficit cognitivo, a partir do ponto de corte sugerido pelos autores acima citados: para aqueles identificados como analfabetos, será de 13; aqueles com escolaridade baixa/média, de 18, e com escolaridade alta, de 26 pontos.

A investigação das quedas foi realizada a partir de um instrumento ${ }^{19}$ que investiga o contexto da queda, causas e consequências que ela pode acarretar ao idoso.

Para avaliação das ABVDs, foi utilizado o Índex de Katz adaptado para o Brasil ${ }^{20}$. Consta de seis itens que medem o desempenho do indivíduo nas atividades de autocuidado, os quais obedecem a uma hierarquia de complexidade, da seguinte forma: alimentação, controle de esfíncteres, transferência, higiene pessoal, capacidade para se vestir e tomar banho. Para a investigação das AIVDs, foi utilizada a Escala de Lawton e Brody, adaptada para o contexto brasileiro ${ }^{21}$.

Os dados foram digitados no programa Microsoft Excel $^{\circledR}$. Após codificação de todas as variáveis em um dicionário, foi elaborado um banco de dados que foi alimentado por técnica de validação por dupla entrada. Concluídas a digitação e a consistência dos dados, estes foram importados no aplicativo Statistical Package for the Social Science (SPSS) for Windows v 19.0.

Todas as variáveis foram submetidas à análise estatística. Para as variáveis qualitativas ou categóricas, medidas de frequência simples; para as variáveis quantitativas, análises empregando-se medidas de tendência central (média e mediana) e dispersão (desvio-padrão). Foi utilizado teste de associação (Teste Quiquadrado) e, para a quantificação da associação, foi utilizada a regressão logística.
O estudo foi desenvolvido garantindo o cumprimento estabelecido na Resolução n ${ }^{\circ}$ 196/96 da Comissão Nacional de Ética e Pesquisa do Ministério da Saúde, tendo sido aprovado pelo Comitê de Ética e Pesquisa da Escola de Enfermagem de Ribeirão Preto da Universidade de São Paulo (EERP/USP), de acordo com o processo $\mathrm{n}^{\mathrm{o}}$ 01852512.6.0000.5393. O Termo de Consentimento Livre e Esclarecido foi elaborado, obedecendo a exigências da Resolução no 196/96 e, antes do início de todas as entrevistas, foi lido e assinado pelo idoso e/ou cuidador/familiar do idoso em duas cópias, das quais uma foi entregue ao entrevistado.

\section{Resultados e Discussão}

Dos 515 idosos visitados, $230(44,6 \%)$ aceitaram participar do estudo e foram entrevistados. Destes, $65,7 \%$ eram do sexo feminino, $42,6 \%$, viúvos e com idade média de 79 anos (70 -103 anos; $d p=6,23)$, a maioria com tempo médio de estudo formal de 1 a 4 anos $(55,8 \%)$ e renda individual mensal de $\mathrm{R} \$ 501,00$ a $\mathrm{R} \$ 1.000,00$ (53,9\%). Ver Tabela 1 .

Entre os entrevistados, $37,8 \%$ sofreram pelo menos uma queda (1-7 quedas; $\mathrm{dp}=1,0)$. Foi mais prevalente no sexo feminino $(25,2 \%)$, em idosos mais jovens (23\%), que vivem sem companheiro $(34,7 \%)$, com 6 a 10 anos de estudo $(23,1 \%)$, com uma renda individual de até $\mathrm{R} \$ 1.000,00$ (21,93\%), que moram com familiares $(21,8 \%)$ e que possuem de 1 a 3 filhos (20\%), conforme mostra a Tabela 1 .

A prevalência de quedas, encontrada no presente estudo, e sua maior ocorrência em mulheres, está em consonância com dados identificados na literatura ${ }^{4,5}$. Em um estudo transversal, realizado com 240 idosos que vivem na comunidade, no Município de Ribeirão Preto (SP), a prevalência de quedas foi de 33,3\%, com maior ocorrência no sexo feminino ${ }^{22}$. Outros estudos nacionais sobre o tema encontraram uma prevalência similar entre $31,2 \%$ e $59,3 \%$, 23 , entre os internacionais em torno de $19,1 \%$ a $31,78 \% 24,25$.

Os dados obtidos ainda demonstraram que as quedas foram mais prevalentes entre os idosos mais jovens (60-79 anos), o que corrobora um estudo nacional $^{22}$, mas difere de outras pesquisas ${ }^{4,26}$ que referem que as quedas são mais prevalentes em idosos mais velhos (80 anos e mais). Esta diferença pode ser atribuída ao fato de os idosos mais jovens serem mais ativos em suas tarefas diárias e, portanto, estarem mais susceptíveis aos fatores de risco relacionados à queda. Pode-se inferir que idosos mais velhos são mais preservados, menos expostos a perigos, em suas atividades diárias. Pode ocorrer maior proteção com relação aos fatores desencadeantes de queda também no que tange ao ambiente e à vestimenta.

Não foi verificada associação entre a queda e a maioria dos dados sociodemográficos investigados. 
Houve associação, apenas, entre quedas e tempo de moradia na mesma casa (p 0,0084). Foi verificado que o idoso que mora no domicílio de 6-10 anos tem 4,6 mais chances de queda (IC-1,566; 13,662), quando comparado aos que moram há 11 anos e mais. É importante ressaltar que, apesar de não ter havido associação entre queda e anos de estudo, foi verificado que a chance de queda vai reduzindo, conforme aumenta o tempo de anos de estudo referidos. $\mathrm{O}$ idoso analfabeto tem uma chance de 2,3 (IC 0,976; 5,448), quando comparado ao que tem seis anos e mais de estudo. $\mathrm{O}$ mesmo ocorreu quando analisada a associação entre queda e renda. Não houve associação, entretanto pôde ser observado que o idoso que não tem renda apresenta uma chance de 2,38 (IC 0,423; 13,387), quando comparado ao que tem renda maior que $\mathrm{R} \$$
$5.000,00$, verificado que quanto maior a renda, menor a chance de queda.

Entre os idosos que sofreram queda, 76,4\% caíram da própria altura, sendo este o tipo de queda mais prevalente, tanto para o sexo masculino $(65,5 \%)$ quanto feminino (81,03\%). Quanto ao local da queda dentro do domicílio, o quintal $(20,0 \%)$ foi o de maior ocorrência e fora do domicílio, a rua (16,25\%).

Em um estudo realizado no Rio de Janeiro, a maioria das quedas registradas ocorreu no domicílio ${ }^{27}$. Em Belo Horizonte, entre os idosos que sofreram queda, 48,44\% caíram da própria altura, e o local de mais ocorrência foi o quintal $(12,5 \%)^{23}$. Na Espanha, investigando 363 idosos, verificou-se que $73,4 \%$ das quedas aconteceram em um lugar conhecido pelo próprio idoso e, dentre estas, 55,3\% aconteceram no domicílio ${ }^{25}$.

TABELA 1: Teste de associação e regressão logística entre queda e variáveis sociodemográficas de idosos que vivem na comunidade. Ribeirão Preto, SP, Brasil, 2013. (N=230)

\begin{tabular}{|c|c|c|c|c|c|}
\hline \multirow[b]{2}{*}{ Variáveis } & \multicolumn{2}{|c|}{ Quedas } & \multirow[b]{2}{*}{ p-valor ${ }^{(*)}$} & \multirow[b]{2}{*}{ Razão } & \multirow[b]{2}{*}{$\begin{array}{c}\text { Intervalo de } \\
\text { Confiança (95\%) }\end{array}$} \\
\hline & $\begin{array}{l}\text { Não } \\
f(\%) \\
\end{array}$ & $\begin{array}{l}\operatorname{Sim} \\
f(\%)\end{array}$ & & & \\
\hline Faixa Etária - 70-79 anos & $82(35,65)$ & $53(23,04)$ & 0,6789 & 1,160 & $(0,673 ; 1,997)$ \\
\hline 80 anos e + & $61(26,52)$ & $34(14,78)$ & & 1,000 & Referência \\
\hline \multicolumn{6}{|l|}{ Sexo } \\
\hline Masculino & $50(21,74)$ & $29(12,61)$ & 0,8863 & 1,000 & Referência \\
\hline Feminino & $93(40,43)$ & $58(25,22)$ & & 1,075 & $(0,612 ; 1,888)$ \\
\hline \multicolumn{6}{|l|}{ Estado civil } \\
\hline c/companheiro & $16(6,96)$ & $7(3,04)$ & 0,5035 & 1,000 & Referência \\
\hline s/companheiro & $127(55,22)$ & $80(34,78)$ & & 1,440 & $(0,567 ; 3,653)$ \\
\hline \multicolumn{6}{|l|}{ Anos estudo } \\
\hline Analfabeto & $20(8,73)$ & $18(7,86)$ & 0,1396 & 2,306 & $(0,976 ; 5,448)$ \\
\hline $1-5$ anos & $82(35,65)$ & $53(23,14)$ & & 1,676 & $(0,855 ; 3,288)$ \\
\hline 6 anos e + & $41(17,90)$ & $16(6,99)$ & & 1,000 & Referência \\
\hline \multicolumn{6}{|l|}{ Renda } \\
\hline Não possui & $7(3,07)$ & $5(2,19)$ & 0,5395 & 2,381 & $(0,423 ; 13,387)$ \\
\hline Até 1000,00 & $73(31,73)$ & $51(22,17)$ & & 2,315 & $(0,606 ; 8,838)$ \\
\hline $1000-5000,00$ & $53(23,25)$ & $28(12,28)$ & & 1,761 & $(0,448 ; 6,924)$ \\
\hline$+5001,00$ & $10(4,39)$ & $3(1,32)$ & & 1,000 & Referência \\
\hline \multicolumn{6}{|l|}{ Tempo moradia na casa } \\
\hline 1-5 anos & $5(2,18)$ & $5(2,18)$ & 0,0084 & 1,928 & $(0,540 ; 6,886)$ \\
\hline 6-10 anos & $5(2,18)$ & $13(5,65)$ & & 4,625 & $(1,566 ; 13,662)$ \\
\hline 11 e mais & $133(58,08)$ & $69(31,13)$ & & 1,000 & Referência \\
\hline \multicolumn{6}{|l|}{ Com quem mora } \\
\hline Sozinho & $31(13,54)$ & $16(6,99)$ & 0,6622 & 1,000 & Referência \\
\hline Somente cônjuge & $39(16,95)$ & $21(9,17)$ & & 1,071 & $(0,479 ; 2,395)$ \\
\hline Familiares & $73(31,88)$ & $50(21,83)$ & & 1,327 & $(0,657 ; 2,679)$ \\
\hline \multicolumn{6}{|l|}{ Pessoas na Casa } \\
\hline Somente idoso & $31(13,48)$ & $15(6,52)$ & 0,5824 & 1,089 & $(0,288 ; 4,114)$ \\
\hline $2-5$ & $103(44,78)$ & $68(29,57)$ & & 1,485 & $(0,440 ; 5,016)$ \\
\hline 5 mais & $9(3,91)$ & $4(1,74)$ & & 1,000 & Referência \\
\hline \multicolumn{6}{|l|}{ Filhos } \\
\hline Nenhum & $18(7,83)$ & $7(3,04)$ & 0,4014 & 1,000 & Referência \\
\hline $1-3$ & $79(34,35)$ & $46(20,00)$ & & 1,497 & $(0,581 ; 3,854)$ \\
\hline Mais de 4 & $46(20,00)$ & $34(14,78)$ & & 1,900 & $(0,714 ; 5,059)$ \\
\hline
\end{tabular}

${ }^{(*)} \mathrm{p}$-valor referente ao Teste Qui-Quadrado 
Os fatores intrínsecos mais prevalentes citados como responsáveis pela queda foram: alteração do equilíbrio $(29,9 \%)$; dificuldade para caminhar $(12,6 \%)$; fraqueza muscular $(11,6 \%)$ e tontura $(6,9 \%)$. Entre os fatores extrínsecos mais citados estão: calçado inadequado $(39,1 \%)$; pisos escorregadios $(17,2 \%)$; pisos irregulares $(10,3 \%)$; degrau alto $(5,7 \%)$ e objetos no chão $(5,7 \%)$.

Este evento multifatorial que contempla dimensões biológica, comportamental, ambiental e socioeconômica envolve aspectos intrínsecos e extrínsecos ${ }^{28-30}$. Evidências na literatura apontam para a existência de, aproximadamente, 400 diferentes fatores de risco para as quedas. Ao menos duas condições de saúde simultâneas e um fator ambiental desempenham papel decisivo na maior parte das ocorrências entre os ido$\operatorname{sos}^{5}$. A prevalência de quedas em idosos que vivem na comunidade e que possuem até um fator de risco para queda é de $27 \%$ e aumenta para $78 \%$ entre aqueles com quatro ou mais fatores associados ${ }^{31,32}$. Assim, subjacente a uma queda, encontram-se a associação entre as disfunções de múltiplos sistemas e órgãos e a influência de aspectos externos ao indivíduo ${ }^{33}$.

As quedas relacionadas a fatores extrínsecos enfatizam a possibilidade de ações educativas a serem implementadas em uma abordagem preventiva. Faz-se necessário modificar os ambientes domésticos de forma a minimizar os perigos, além de promover a saúde, prevenir doenças e incapacidades do idoso com o objetivo de diminuir os riscos que possam propiciar quedas ${ }^{22}$.

Observou-se que, entre os idosos que caíram, do presente estudo, a consequência principal citada foi medo de voltar a cair, seguida por afetou o andar, hospitalização e necessidade de ajuda para as atividades. Essas consequências estão em consonância com resultados de outros estudos. Em âmbito mundial, as quedas são responsáveis por $87 \%$ das fraturas e $50 \%$ das internações dos idosos ${ }^{3}$. Nos Estados Unidos, $25,6 \%$ da amostra, constituída por 5.681 idosos, caiu, e, entre estes, $61 \%$ apresentaram algum tipo de lesão e $20 \%$ foram hospitalizados ${ }^{34}$. No Brasil, um estudo identificou em 2.209 idosos (com idade $\geq 60$ anos) que vivem no domicílio uma prevalência de $27,1 \%$ de quedas e $8,7 \%$ para quedas recorrentes ${ }^{35}$. Em outra pesquisa realizada com 6.616 idosos brasileiros com 65 anos e mais, constatou-se uma frequência de $27,6 \%$ de quedas, $11 \%$ resultaram em fratura e $1 \%$ em atendimento cirúrgico ${ }^{36}$.

A literatura descreve que os idosos, em geral, têm baixo senso de eficácia para evitar a queda, apresentam ainda medo de cair, restrição em atividades e perda de confiança em si mesmos ${ }^{37}$. A síndrome pós-queda e o medo de cair afetam 73\% daqueles que sofreram o evento no ano anterior, já, entre os idosos sem relato de quedas recentes, a prevalência foi de $46 \%{ }^{38}$. Dessa forma, o medo de cair pode resultar em uma síndrome pós-queda que inclui dependência, perda de autonomia, imobilização, isolamento e depressão, o que levará a uma maior restrição nas tarefas diárias ${ }^{39}$.

A independência, entendida como a habilidade de executar funções relacionadas à vida diária (ABVDs e AIVDs) de maneira autônoma, com pouco ou nenhuma ajuda de outros, envolve capacidade funcional. Ela permite que o indivíduo empreenda ações relativas às suas decisões sobre como se deve viver diariamente, de acordo com suas próprias regras e preferências ${ }^{16}$.

No presente estudo, não foi verificada associação entre queda e AIVDs, conforme verificado na Tabela 2. Entretanto, há evidências de que os idosos com independência $(23,48 \%)$ foram mais prevalentes entre os que não caíram e a dependência total (2,61\%) entre os que sofreram queda. Ainda, foi verificado que idosos com dependência total apresentaram 2,314 $(0,649 ; 8,253)$ mais chances de cair, quando comparados aos idosos com independência.

Com relação às ABVDs, foi verificada sua associação com quedas. Ainda, os idosos com dependência total possuíam uma chance de 4,498 (IC 1,239; 16,324) de queda, quando comparados aos que eram independentes para estas atividades, ou seja, quanto maior a dependência para estas atividades, maior o risco de queda. Entre as atividades investigadas na avaliação das ABVDs, as mais associadas à queda foram continência e uso do vaso sanitário.

É possível inferir que este achado pode ter relação com o fato de as AIVDs serem mais complexas e elaboradas e exigirem habilidades cognitivas com maior intensidade comparadas às motoras. Assim, as AIVDs podem ser atividades menos executadas pelos idosos, quando comparadas às ABVDs.

A avaliação multidimensional do idoso possibilita tanto identificar as incapacidades e limitações como as necessidades de cuidados, direcionando as intervenções ${ }^{40}$. Este tipo de avaliação enfatiza a funcionalidade da pessoa idosa. Nela, é necessário incluir a história de saúde do indivíduo, a avaliação da função física, a análise de componentes sensoriais (audição e visão), medidas de sinais ortostáticos e neurológicos, avaliação musculoesquelética, disfunção cognitiva e depressão ${ }^{14}$.

A avaliação de AVDs pode auxiliar nesta investigação multidimensional do idoso, pois as ABVDs podem ser um forte indicador de que o idoso necessita de maior atenção e cuidados relacionados à prevenção de quedas. Infere-se que promover e recuperar a autonomia e independência do idoso na execução destas atividades pode ser uma estratégia de prevenção de quedas. Entretanto, esta indicação necessita ser mais investigada em estudos de coorte que quantifiquem o quanto esta estratégia pode ser efetiva para esta prevenção. 
TABELA 2: Teste de associação e razão de chances entre quedas, AIVDs e ABVDs em idosos que vivem na comunidade. Ribeirão Preto, SP, Brasil, 2013. ( $\mathrm{N}=230)$

\begin{tabular}{|c|c|c|c|c|c|}
\hline \multirow[t]{2}{*}{ Variáveis } & \multicolumn{2}{|c|}{ Quedas } & \multirow[b]{2}{*}{ p-valor ${ }^{(*)}$} & \multirow[b]{2}{*}{ Razão } & \multirow[b]{2}{*}{$\begin{array}{c}\text { Intervalo de } \\
\text { Confiança (95\%) }\end{array}$} \\
\hline & $\begin{array}{l}\text { Não } \\
\mathrm{f}(\%)\end{array}$ & $\begin{array}{l}\text { Sim } \\
f(\%)\end{array}$ & & & \\
\hline \multicolumn{6}{|l|}{ AIVDs } \\
\hline Independência & $54(23,48)$ & $28(12,17)$ & 0,402 & 1,000 & Referência \\
\hline Dependência Parcial & $84(36,52)$ & $53(23,04)$ & & 1,217 & $(0,687 ; 2,155)$ \\
\hline Dependência Total & $5(2,17)$ & $6(2,61)$ & & 2,314 & $(0,649 ; 8,253)$ \\
\hline \multicolumn{6}{|l|}{ Banho } \\
\hline Independência & $133(57,83)$ & $75(32,61)$ & 0,2333 & 1,000 & Referência \\
\hline Dependência Parcial & $9(3,91)$ & $11(4,78)$ & & 2,167 & $(0,859 ; 5,467)$ \\
\hline Dependência Total & $1(0,43)$ & $1(0,43)$ & & 1,773 & $(0,109 ; 28,763)$ \\
\hline \multicolumn{6}{|l|}{ Vestir } \\
\hline Independência & $134(58,26)$ & $75(32,61)$ & 0,0555 & 1,000 & Referência \\
\hline Dependência Parcial & $9(3,91)$ & $12(5,22)$ & & 2,382 & $(0,959 ; 5,913)$ \\
\hline \multicolumn{6}{|l|}{ Uso Vaso sanitário } \\
\hline Independência & $136(59,13)$ & $76(33,04)$ & 0,0339 & 1,000 & Referência \\
\hline Dependência Parcial & $7(3,04)$ & $11(4,78)$ & & 2,811 & $(1,046 ; 7,554)$ \\
\hline \multicolumn{6}{|l|}{ Transferência } \\
\hline Independência & $132(57,39)$ & $75(32,61)$ & 0,1347 & 1,000 & Referência \\
\hline Dependência Parcial & $11(4,78)$ & $12(5,22)$ & & 1,920 & $(0,808 ; 4,564)$ \\
\hline \multicolumn{6}{|l|}{ Continência } \\
\hline Independência & $97(42,17)$ & $38(16,52)$ & 0,0003 & 1,000 & Referência \\
\hline Dependência Parcial & $46(20,00)$ & $49(21,30)$ & & 2,719 & $(1,568 ; 4,712)$ \\
\hline \multicolumn{6}{|l|}{ Alimentação } \\
\hline Independência & $139(60,43)$ & $81(35,22)$ & 0,1393 & 1,000 & Referência \\
\hline Dependência Parcial & $4(1,74)$ & $6(2,61)$ & & 2,574 & $(0,705 ; 9,391)$ \\
\hline \multicolumn{6}{|l|}{ ABVDs } \\
\hline Independência & $90(39,13)$ & $35(15,22)$ & 0,0022 & 1,000 & Referência \\
\hline Dependência Parcial & $49(21,30)$ & $45(19,57)$ & & 2,361 & $(1,346 ; 4,143)$ \\
\hline Dependência Total & $4(1,74)$ & $7(3,04)$ & & 4,498 & $(1,239 ; 16,324)$ \\
\hline
\end{tabular}

${ }^{(*)} \mathrm{p}$-valor referente ao Teste Qui-Quadrado

\section{CONCLUSÃO}

Os dados identificados reforçam a importância de se conhecer o contexto da queda, da identificação de fatores envolvidos neste evento e das consequências que ela pode acarretar, para delinear estratégias preventivas frente a este evento tão frequente entre os idosos.

A principal limitação deste trabalho foi o fato de se tratar de um estudo transversal, o que impossibilita a ordem de causalidade entre as variáveis. Entretanto, o percentual de resposta e o fato de a amostra ser derivada de um estudo de base populacional aumentam a relevância do trabalho.

Perante as evidências desta pesquisa, sugere-se que a investigação das ABVDs seja utilizada como estratégia para a prevenção de quedas. Uma vez identificada deficiência na execução destas atividades, é necessário utilizar intervenções que recuperem e promovam a independência do idoso para promoção da autonomia e independência.
A queda nos idosos é um evento complexo e multifatorial, cuja prevenção se reporta como necessária e ainda se constitui em um desafio para o próprio idoso, familiares, profissionais de saúde e políticas públicas.

\section{REFERÊNCIAS}

1.Organización de Las Naciones Unidas. Informe de la segunda asemblea mundial sobre el envejecimiento. Madrid (Es): Naciones Unidas; 2002.

2.Cruz DT, Ribeiro LC, Vieira MT, Teixeira MTB, Bastos RR., Leite ICCG. Prevalence of falls and associated factors in elderly individuals. Rev Saude Publica. 2012; 46:38-46. 3. World Heath Organization (WHO). World Heath Organization Report: prevention of falls in older age. Geneva (Swi): WHO; 2007.

4.Ciaschini PM, Straus SE, Dolovich LR, Goeree RA, Leung KM, Woods CR, et al. Community-based intervention to optimise falls risk management: a randomised controlled trial. Age and Ageing. 2009; 38:724-30.

5.Czerwinski E, Białoszewski D, Borowy P, Kumorek A, Białoszewski A. Epidemiology, clinical significance, costs and 
fall prevention in elderly people. Ortop Traumatol Rehabil. 2008; 10:419-28.

6.Kwan MM, Close JCT, Wong AKW, Lord SR. Falls incidence, risk factors, and consequences in chinese older people: a systematic review. JAGS. 2011; 59:536-43.

7.Goins RT, Innes K, Dong L. Lower body functioning prevalence and correlates in older american indians in a southeastern tribe: the native elder care studs. J Am Geriatr Soc. 2012; 60:577-82.

8. Wang J, Chen Z, Song Y. Falls in aged people of the Chinese mainland: epidemiology, risk factors and clinical strategies. Ageing Res Reviews. 2010;13-7.

9.Bleijlevens MHC, Diederiks JPM, Hendriks MRC, van Haastregt JCM, Crebolder HFJM, van Eijk JTHM. Relationship between location and activity in injurious falls: an exploratory study. BMC Geriatrics. 2010; 10:40.

10.Langlois F, Vu TTM, Kergoat M, Chassé K, Dupuis G, Bherer L. The multiple dimensions of frailty: physical capacity, cognition, and quality of life. Int Psychogeriatrics. 2012; 24:1429-36.

11.Zijlstra GAR, van Haastregt JCM, van Eijk JTM, van Rossum E, Stalenhoef PA, Kempen GIJM. Prevalence and correlates of fear of falling, and associated avoidance of activity in the general population of community-living older people. Age and Ageing. 2007; 36:304-9.

12.Ribeiro AP, Souza ER, Atie S, Souza AC, Schilithz AO. A influência das quedas na qualidade de vida de idosos. Ciênc saúde coletiva. 2008; 13:1264-73.

13.Freiberger E, Vreede P. Falls recall: limitations of the most used inclusion criteria. Eur Rev Aging Phys Act. 2011; 8:105-8.

14.Al-Aama T. Falls in the elderly: spectrum and prevention. Can Fam Physician 2011; 57:771-6.

15.Antes DL, Schneider IJC, Benedetti TRB, D'orsi E. Medo de queda recorrente e fatores associados em idosos de Florianópolis, Santa Catarina, Brasil. Cad Saúde Pública. 2013; 29:758-68.

16.Silva MJ, Lopes MVO, Araujo MFM, Morales GLA. Avaliação do grau de dependência nas atividades da vida diária em idosos da cidade de Fortaleza - Ceará. Acta Paul Enferm. 2006; 19:201-6.

17.Fiedler MM, Peres KG. Capacidade funcional e fatores associados em idosos do Sul do Brasil: um estudo de base populacional. Cad Saúde Pública. 2008; 24:409-15

18.Bertolucci PHF, Brucki SMD, Campacci SR., JulianoY. O mini exame do estado mental em uma população geral. Impacto da escolaridade. Arq Neuropsiquiatr.1994; 52:1-7. 19.Schiaveto FV. Avaliação do risco de quedas em idosos na comunidade [dissertação de mestrado]. Ribeirão Preto (SP): Universidade de São Paulo; 2008.

20.Lino VTS, Pereira SRM, Camacho LAB, Ribeiro FST, Buksman S. Adaptação transcultural da Escala de Independência em atividades da vida diária (escala de Katz). Cad Saúde Pública. 2008; 24:103-12.

21.Santos RL, Virtuoso Jr JS. Confiabilidade da versão brasileira da escala de atividades instrumentais da vida diária. RBPS. 2008; 21:290-6.

22.Fhon JRS, Fabrício-Wehbe SCC, Vendruscolo TRP, Stackfleth R, Marques S, Rodrigues RAP. Accidental falls in the elderly and their relation with functional capacity. Rev Latino-Am Enfermagem. 2012; 20:927-34.

23.Chianca TCM, Andrade CR, Albuquerque J, Wenceslau LCC, Tadeu LFR, Macieira TGR, et al. Prevalence of fall in older adults enrolled in a health center of Belo Horizonte MG. Rev Bras Enferm. 2013; 66:234-40.

24.Skalska A, Wizner B, Piotrowicz K, Klich-Raczka A, Klimek E, Mossakowska M, et al. The prevalence of falls and their relation to visual and hearing impairments among a nation-wide cohort of older poles. Experimental Gerontology. 2013; 48:140-6.

25.Varas-Fabra F, Castro ME, Pérula TLA, Fernández FMJ, Moral RR, Enciso BI. Caídas em ancianos de la comunidad: prevalencia, consecuencias y factores asociados. Aten Primaria. 2006; 38:450-5.

26.Nicolussi AC, Fhon JRS, Santos CAV, Kusumota L, Marques S, Rodrigues RAP. Quality of life in elderly people that have suffered falls: integrative literature review. Ciênc saúde coletiva. 2012; 17:723-30.

27.Coutinho ESF, Bloch KV, Rodrigues LC. Characteristics and circumstances of falls leading to severe fractures in elderly people in Rio de Janeiro, Brazil. Cad Saúde Pública. 2009; 25:455-9.

28.Almeida ST, Soldera CLC, Carli GA, Gomes I, Resende L. Análise de fatores extrínsecos e intrínsecos que predispõem a quedas em idosos. Rev Assoc Med Bras. 2012; 58:427-33. 29.Fox PJ, Vazquez L, Tonner C, Stevens JA, Fineman N, Ross LK. A randomized trial of a multifaceted intervention to reduce falls among community-dwelling adults. Health Education Behavior. 2010; 37:831-48.

30.Stevens JA, Baldwin GT, Ballesteros MF, Noonan RK, Sleet DA. An older adult falls research agenda from a public health perspective. Clin Geriatr Med. 2010; 26:767-79.

31.American Geriatrics Society and British Geriatrics Society (AGS e BGS). Summary of the updated American Geriatrics Society/British Geriatrics Society. Clinical practice guideline for prevention of falls in older persons. New York: AGS/ BGS; 2010.

32.Tinetti ME, Kumar C. The patient who falls: "it's always a trade-off." JAMA. 2010; 303:258-66.

33.Gschwinda YJ, Wolfa I, Bridenbaugha SA, Kressig RW. Basis for a Swiss perspective on fall prevention in vulnerable older people. Swiss Med Wkly. 2011; 141:w13305, 2011.

34.Milat JA, Watson WL, Monger C, Barr M, Giffin M, Reid M. Prevalence, circumstances and consequences of falls among community-dwelling older people: results of the 2009 NSW Falls Prevention Baseline Survey. NSW Public Health Bulletin. 2011; 22:43-8.

35.Coimbra AMV, Ricci NA, Coimbra IB, Costallat LTL. Falls in the elderly of the family health program. Arch Gerontol Geriatr. 2010; 51:317-22.

36.Siqueira FV, Facchini LA, Silveira DS, Piccini RX, Tomasi E, Thumé E, et al. Prevalência de quedas em idosos no Brasil: uma análise nacional. Cad Saúde Pública. 2011; 27:1819-26. 37.Scheffer AC, Schuurmans MJ, Dijk NV, Hooft TV, Rooij SE. Fear of falling: measurement strategy, prevalence, risk factors and consequences among older persons. Age and Ageing. 2008; 37:19-24.

38.Fabre JM, Ellis R, Kosma M, Wood R.H. Falls risk factors and a compendium of falls risk screening instruments. J Geriatr Phys Ther. 2010; 33:184-97.

39. Organização Mundial Da Saúde. Relatório global da OMS sobre prevenção de quedas na velhice. São Paulo: Secretaria de Estado da Saúde São Paulo; 2010.

40.Costa AGS, Costa FBC, Oliveira ARS, Silva VM, Araujo TL. Ocorrência de quedas e índice de massa corporal em idosos. Rev enferm UERJ. 2013; 21:508-14. 